\title{
THE ISOMORPHIC REFINEMENT THEOREM FOR DIRECT SUMS OF CLOSED GROUPS ${ }^{1}$
}

PAUL HILL

The purpose of this paper is to prove that if the primary group $G$ is a direct sum of closed groups, then any two direct decompositions of $G$ have isomorphic refinements. Special cases of this theorem have been proven by Kulikov [10], Kolettis [9], Enochs [1], and Irwin, Richman and Walker [8]. A special case due to Kulikov is now considered classical: If the primary group $G$ is a direct sum of cyclic groups, then any two direct decompositions of $G$ have isomorphic refinements. This result yields rather immediately the following, also due to Kulikov [10]. If $G$ is a closed group, then any two direct decompositions of $G$ admit isomorphic refinements. In [9] Kolettis embedded the two theorems of Kulikov into a slightly more general result. Enochs proved in [8] that any two direct decompositions of a primary group $G$ in to closed groups have isomorphic refinements, but it was not known heretofore that a direct summand of a direct sum of closed groups is always itself a direct sum of closed groups. However, it was shown in [1] that a direct summand of a direct sum of a countable number of closed groups is necessarily a direct sum of closed groups, and the general isomorphic refinement theorem was established for a group $G$ that can be expressed as a direct sum of a countable number of a closed group. As indicated above, in this paper we shall remove the countability hypothesis.

We follow, for the most part, the notation and terminology of [2]. In particular, we use the term "closed" instead of "torsion complete."

Lemma 1. Suppose that $H$ is a closed group and that $G=\sum_{\lambda \in \Lambda} G_{\lambda}$ is a direct sum of closed groups $G_{\lambda}$. If $\phi$ is a homomorphism from $H[p]$ into $G$ that does not decrease heights (computed in $H$ and $G$ ), then there is a positive integer $n$ and a finite subset $M$ of $\Lambda$ such that $\phi\left(p^{n} H \cap H[p]\right)$ $\subseteq \sum_{\lambda \in M} G_{\lambda}$.

Proof. Although the proof of Lemma 1 requires only a slight modification of the proof of similar but weaker results in [1], [5] and [8], we include an outline of the proof for completeness. If the lemma were false, we could choose $x_{n} \in\left(p^{k} H \cap H[p]\right)$ such that:

(1) $p^{k} G \cap\left\{\pi_{\lambda} \phi\left(x_{1}\right), \pi_{\lambda} \phi\left(x_{2}\right), \cdots, \pi_{\lambda} \phi\left(x_{n-1}\right)\right\}_{\lambda \in \Lambda}=0$ where $\pi_{\lambda}$ is the natural projection of $G$ on to $G_{\lambda}$.

Received by the editors August 10, 1966.

1 This research was supported by NSF Grant GP-5875. 
(2) $k \geqq n$, and

(3) $\phi\left(x_{n}\right) \notin \sum_{\lambda \in M} G_{\lambda}$ where $M$ is the minimal subset of $\Lambda$ such that $\left\{\phi\left(x_{1}\right), \phi\left(x_{2}\right), \cdots, \phi\left(x_{n-1}\right)\right\} \subseteq \sum_{\lambda \in M} G_{\lambda}$. Now $\sum x_{n}$ converges in $H$, but $\sum \phi\left(x_{n}\right)$ does not converge in $G$; hence $\phi$ is discontinuous (in the $p$-adic topology) but this is impossible since $\phi$ does not decrease heights.

The next lemma states a rather immediate corollary of the preceding one.

Lemma 2. Suppose that the primary group $H$ is a closed group and that $G=\sum\left\{g_{\lambda}\right\}$ is a direct sum of cyclic groups. If $\phi$ is a homomorphism from $H[p]$ into $G$ that does not decrease heights, then there is a positive integer $n$ such that $\phi\left(p^{n} H \cap H[p]\right)=0$.

Lemma 1 and Lemma 2 combine readily to yield the following result, which is what we actually need in the sequel.

LeмMA 3. Suppose that $H$ is a closed group and that $G=B+\sum_{\lambda \in \Delta} G_{\lambda}$ where $B$ is a direct sum of cyclic groups and $G_{\lambda}$ is closed for each $\lambda \in \Lambda$. If $\phi$ is a homomorphism from $H[p]$ into $G$ that does not decrease heights, then there is a positive integer $n$ and a finite subset $M$ of $\Lambda$ such that $\phi\left(p^{n} H \cap H[p]\right) \subseteq \sum_{\lambda \in M} G_{\lambda}$.

By a projection of a group $G$, we mean an idempotent endomorphism of $G$. It is an elementary and well-known result that a subgroup $H$ of an abelian group $G$ is a direct summand of $G$ if and only if $H=\phi(G)$ is the image of some projection $\phi$ of $G$. Moreover, if a subsocle $S$ of the primary group $G$ supports a direct summand of $G$, then $S$ must be the image of a projection of $G[p]$ that does not decrease heights; however, it follows from [3] and [11] that the converse does not hold. That the converse is valid for pure-complete groups $G$ is the content of Theorem 1. Recall that a pure-complete group is a primary group with the property that each subsocle supports a pure subgroup; see [4] and [7].

THEOREM 1. If the primary group $G$ is pure-complete and if $\phi$ is a projection of $G[p]$ that does not decrease heights, then the image of $\phi$ supports a direct summand of $G$.

Proof. Let $H$ be a pure subgroup of $G$ such that $H[p]=\phi(G[p])$ and let $S$ be the subsocle of $G+H$ defined by $S=\{(x, \phi(x)): x \in G[p]\}$. The subgroup $K=\{(h, h): h \in H\}$ is a pure subgroup of $G+H$. Since $(G+H) / K$ is isomorphic to $G$, it is pure-complete. Let $L / K$ be a pure subgroup of $(G+H) / K$ such that $(L / K)[p]=\{S, K\} / K$. Then $L$ is a pure subgroup of $G+H$ and $L[p]=S$. Since $L[p]=S$, it is clear that 
$L$, as a subgroup of $G+H$, has trivial intersection with $H$. Since $\phi$ does not decrease heights and since $L$ is pure, we can show by induction on $n$ that $G\left[p^{n}\right] \subseteq\{L, H\}$. Thus $G \subseteq\{L, H\}$ and the natural projection of $G+H$ on to $H$ is a projection of $G$ on to $H$ when restricted to $L$ if $G$ and $L$ are identified in the obvious way.

COROLLARY 1. If the primary group $G$ is a direct sum of a countable number of closed groups and if $S$ is a subsocle of $G$, then $S$ supports a direct summand of $G$ if and only if $S$ is the image of a projection of $G[p]$ that does not decrease heights.

Proof. A direct sum of a countable number of closed groups is pure-complete by Theorem 5.6 in [7].

Since a primary group $G$ is a direct sum of a countable number of closed groups if it is the direct sum of any number of cyclic groups, we have

COROLlaRY 2. If the primary group $G$ is a direct sum of cyclic groups and if $S$ is a subsocle of $G$, then $S$ supports a direct summand of $G$ if and only if $S$ is the image of a projection of $G[p]$ that does not decrease heights.

The next theorem is of a technical nature, but our main result rests heavily upon it.

THEOREM 2. For the primary group $G$, suppose that $G=\sum_{\lambda \in \Delta} G_{\lambda}$ where $G_{\lambda}$ is closed for each $\lambda \in \Lambda$. Suppose that $\phi$ is a projection of $G[p]$ that does not decrease heights. Then $G=B+\sum_{\mu \in M} H_{\mu}$ where $B$ is a direct sum of cyclic groups, $M \subseteq \Lambda, H_{\mu}$ is closed for each $\mu \in M, \phi\left(H_{\mu}[p]\right)$ $\subseteq \sum_{\nu \in N} H_{\nu}$ for some finite subset $N=N_{\mu}$ of $M$, and $\phi(B[p]) \subseteq B$.

Proof. The theorem is trivial in case $\Lambda$ is finite; take $B=0, M=\Lambda$, $H_{\lambda}=G_{\lambda}$. In order to free ourselves from a few technicalities, we shall assume that $\Lambda$ is uncountable and, in the end, observe that with an obvious modification the proof covers the countable case as well.

Choose the index set $\Lambda$ to be the initial segment of ordinal numbers less than $\Omega$ where $\Omega$ is the smallest ordinal of cardinality $m>N_{0}$. Suppose that $\gamma \in \Lambda$ and that, for each $\beta<\gamma$, we have chosen a subset $I_{\beta}$ of $\Lambda$ and subset $M_{\beta}$ of $I_{B}$ such that:

(1) $\sum_{I_{\beta}} G_{\lambda}=\sum_{M_{\beta}} B_{\mu}+\sum_{M_{\beta}} H_{\mu}$, where $B_{\mu}$ is a direct sum of cyclic groups and $H_{\mu}$ is closed for each $\mu \in M_{\beta}$.

(2) If $\alpha<\beta<\gamma$, then $I_{\alpha} \subseteq I_{\beta}$ and $M_{\alpha} \subseteq M_{\beta}$. In the decomposition (1), $B_{\mu}$ and $H_{\mu}$ are independent of $\beta$.

(3) For each $\mu \in M_{\beta}, \phi\left(H_{\mu}[p]\right) \subseteq \sum_{N} H_{\nu}$ for some finite subset $N$ of $M_{\beta}$. 
(4) $\beta \in I_{\beta+1}$.

(5) $\left|I_{\beta}\right| \leqq \aleph_{0}|\beta|$ and $I_{0}=\varnothing$.

If $\gamma$ is a limit ordinal, define $I_{\gamma}=\bigcup_{\beta<\gamma} I_{\beta}$ and $M_{\gamma}=\bigcup_{\beta<\gamma} M_{\beta}$. Then conditions (1) $-(5)$ hold for $\beta \leqq \gamma$.

Assume that $\gamma-1$ is defined. Suppose that $n$ is a positive integer and that $\varnothing=J_{0} \subset J_{1} \subset \cdots \subset J_{n}$ is a strictly ascending sequence of finite subsets of $\Lambda$ such that the following conditions hold. Set $K(i)=J_{i}-J_{i-1}$ for $i=1,2, \cdots, n$.

(i) $J_{n} \cap I_{\gamma-1}=\varnothing$.

(ii) If $1 \leqq i<n$, then $\sum_{K(i)} G_{\gamma}=B_{\mu(i)}+H_{\mu(i)}$ where $B_{\mu(i)}$ is bounded and $\mu(i)$ is the first element of $K(i)$.

(iii) If $1 \leqq k<n-1$, then $\phi\left(H_{\mu(k)}[p]\right) \subseteq \sum_{F} H_{\mu}+\sum_{1}^{k+1} H_{\mu(i)}$ where $F$ is a finite subset of $M_{\gamma-1}$.

(iv) $\phi\left(H_{\mu(n-1)}[p]\right) \subseteq \sum_{F} H_{\lambda}+\sum_{1}^{n-1} H_{\mu(i)}+\sum_{K(n)} G_{\lambda}$ where $F$ is a finite subset of $M_{\gamma-1}$.

Note that we may assume that $\gamma-1 \in\left(I_{\gamma-1} \cup J_{n}\right)$. We wish to define a finite subset $J_{n+1}$ of $\Lambda$ in such a way that conditions (i)-(iv) remain valid when $n+1$ replaces $n$. Throughout the remainder of the proof, we shall reserve the symbol $\sum_{F}$ to denote a finite summation over $M_{\gamma-1}$, that is, $F$ is understood to denote an arbitrary (not fixed) finite subset of $M_{\gamma-1}$.

Since $K(n)=J_{n}-J_{n-1}$ is finite, $\sum_{K(n)} G_{\lambda}$ is closed. It follows from Lemma 3 that there is a finite set $L$ of $\Lambda$ and a decomposition

(I) $\sum_{K(n)} G_{\lambda}=B_{\mu(n)}+H_{\mu(n)}$ such that $B_{\mu(n)}$ is bounded, $L \cap$ $\left(I_{\gamma-1} \cup J_{n}\right)=\varnothing$, and

(II) $\phi\left(H_{\mu(n)}[p]\right) \subseteq \sum_{F} H_{\mu}+\sum_{1}^{n} H_{\mu(i)}+\sum_{L} G_{\lambda}$.

By condition (5), we may assume that $L$ is nonempty. And since $K(n)$ is nonempty, we can choose $\mu(n)$ to be the first element of $K(n)$; this is an admissible index for $B$ and $H$ in the sense that is has not been used before.

Now a crucial point of the proof is that the decomposition (I) can be made in such a way that it enjoys an additional property:

(III) $\phi\left(H_{\mu(n-1)}[p]\right) \subseteq \sum_{F} H_{\mu}+\sum_{1}^{n} H_{\mu(i)}$. We know, by condition (iv), that

(IV) $\phi\left(H_{\mu(n-1)}[p]\right) \subseteq \sum_{F} H_{\mu}+\sum_{1}^{n-1} H_{\mu(i)}+B_{\mu(n)}+H_{\mu(n)}$.

Since $B_{\mu(n)}$ is bounded, we can write $B_{\mu(n)}=A_{\mu(n)}+C_{\mu(n)}$ where $C_{\mu(n)}[p]$ is the image of $\phi\left(H_{\mu(n-1)}[p]\right)$ under the natural projection of $\sum_{M_{\gamma-1}} H_{\mu}+\sum_{1}^{n-1} H_{\mu(i)}+B_{\mu(n)}+H_{\mu(n)}$ onto $B_{\mu(n)}$. Suppose that $x \in C_{\mu(n)}[p]$. For some $y \in H_{\mu(n-1)}[p]$,

$$
\phi(y)=h+h_{1}+h_{2}+\cdots+h_{n-1}+x+h_{n}
$$

where $h \in \sum_{F} H_{\mu}$ and $h_{i} \in H_{\mu(i)}$ for $i=1,2, \cdots, n$. Since $\phi^{2}=\phi$, we have 


$$
\phi\left(y-h_{n-1}\right)=\phi(h)+\phi\left(h_{1}\right)+\cdots+\phi\left(h_{n-2}\right)+\phi(x)+\phi\left(h_{n}\right) .
$$

Thus $\phi(x) \in \sum_{F} H_{\mu}+\sum_{1}^{n-1} H_{\mu(i)}+C_{\mu(n)}+H_{\mu(n)}+\sum_{L} G_{\lambda}$. It follows that

$$
\phi\left(\left(C_{\mu(n)}+H_{\mu(n)}\right)[p]\right) \subseteq \sum_{F} H_{\mu}+\sum_{1}^{n-1} H_{\mu(i)}+C_{\mu(n)}+H_{\mu(n)}+\sum_{L} G_{\lambda} .
$$

Thus we may replace $H_{\mu(n)}$ by $C_{\mu(n)}+H_{\mu(n)}$ and $B_{\mu(n)}$ by $A_{\mu(n)}$ and, in the process, gain condition (III).

Define $J_{n+1}=J_{n} \cup L$, and the induction is completed on the sequence $\left\{J_{n}\right\}$. Defining $I_{\gamma}=I_{\gamma-1} \cup\left(\bigcup_{n<\omega} J_{n}\right)$ and $M_{\gamma}=\left\{M_{\gamma-1}, \mu(n)\right\}_{n<\omega}$, we have at the same time completed the induction on the existence of the chains $\left\{I_{\lambda}\right\}$ and $\left\{M_{\lambda}\right\}, \lambda \in \Lambda$, with the desired properties (1)(5). We remark that in case $\Lambda$ is countable we could exhaust $\Lambda$, with the proper choice of $J_{n}$, in one step, that is, with $I_{1}$ and the argument simplifies.

Clearly, the $I_{\lambda}$ 's and $M_{\lambda}$ 's satisfying conditions (1)-(5) yield a decomposition $G=B+\sum_{\mu \in M} H_{\mu}$ that satisfies all the conditions of the theorem except, possibly, the condition $\phi(B[p]) \subseteq B$. Let $\rho$ and $\sigma$ denote the projections associated with the decomposition $G=B$ $+\sum_{\mu \in M} H_{\mu}$, where $\rho(G)=B$. The endomorphism $\rho \phi$ of $B[p]$ is actually a projection of $B[p]$ that does not decrease heights. Corollary 2 implies that $\rho \phi(B[p])$ supports a direct summand of $B$. Let $B=B_{0}$ $+B_{1}$ where $B_{1}[p]=\rho \phi(B[p])$. Define $S_{0}=(1-\phi)\left(B_{0}[p]\right)$ and $S_{1}$ $=(1+\sigma \phi) B_{1}[p]$. There are pure subgroups $B_{0}^{\prime}$ and $B_{1}^{\prime}$ of $G$ such that $B_{0}^{\prime}[p]=S_{0}, B_{1}^{\prime}[p]=S_{1}, \rho\left(B_{0}^{\prime}+B_{1}^{\prime}\right)=B_{0}+B_{1}$. Letting $B^{\prime}=B_{0}^{\prime}+B_{1}^{\prime}$, we have that $G=B^{\prime}+\sum_{\mu \in M} H_{\mu}$. It is straightforward to show that $\phi\left(B^{\prime}[p]\right) \subseteq B^{\prime}$, and the proof of Theorem 2 is finished.

We are now prepared to prove a result which contains as a corollary the fact that a direct summand of a direct sum of closed groups is a direct sum of closed groups.

ThEOREM 3. Suppose that the primary group $G=\sum_{\lambda \in \Delta} G_{\lambda}$ is a direct sum of closed groups $G_{\lambda}$. If $\phi$ is a projection of $G[p]$ that does not decrease heights, then $S=\phi(G[p])$ supports a direct summand of $G$. Furthermore, if $H$ is any such direct summand of $G$, then $H=\sum_{\lambda \in \Lambda} H_{\lambda}$ where $H_{\lambda}$ is a closed group.

Proof. The proof is by induction on the cardinality of $\Lambda$. If $\Lambda$ is countable, Corollary 1 states that $S$ supports a direct summand of $G$. A direct summand of $G$, in case $\Lambda$ is countable, is a direct sum of a countable number of closed groups by Theorem 1 in [8]. Thus we may assume that $\Lambda$ is uncountable. By Theorem 2, there is no loss of generality in assuming that, for each $\lambda \in \Lambda$, there is a countable sub- 
set $M$ of $\Lambda$ such that $\phi\left(G_{\lambda}[p]\right) \subseteq \sum_{\mu \in M} G_{\mu}$. Taking $\Lambda$ to be the initial segment of ordinal numbers less than $\Omega$ where $\Omega$ is the least ordinal of cardinality $|\Lambda|$ and using an argument similar to that employed in [6], we easily obtain an ascending chain

$$
I_{0} \subseteq I_{1} \subseteq I_{2} \subseteq \cdots \subseteq I_{\lambda} \subseteq \cdots, \quad \lambda \in \Lambda,
$$

of subsets of $\Lambda$ such that $I_{0}=\varnothing, \lambda \in I_{\lambda+1}, I_{\lambda}=U_{\mu<\lambda} I_{\mu}$ if $\lambda$ is a limit ordinal, $\left|I_{\lambda}\right| \leqq \boldsymbol{\aleph}_{0}|\lambda|$, and $\phi\left(\sum_{I_{\lambda}} G_{\mu}[p]\right) \subseteq \sum_{I_{\lambda}} G_{\mu}$ for each $\lambda \in \Lambda$.

Suppose that $\sum_{I_{\lambda}} G_{\mu}=H+K$ where $\phi\left(\sum_{I_{\lambda}} G_{\mu}[p]\right)=H[p]$. Since $\left|I_{\lambda}\right| \leqq \aleph_{0}|\lambda|<|\Lambda|$, the induction hypothesis implies that $K=\sum_{I_{\lambda}} K_{\mu}$ where $K_{\mu}$ is closed. Now

$$
\sum_{I_{\lambda+1}} G_{\mu}=H+\left(\sum_{I_{\lambda}} K_{\mu}+\sum_{I_{\lambda}+1-I_{\lambda}} G_{\mu}\right)
$$

Let $\pi$ be the projection of $\sum_{I_{\lambda+1}} G_{\mu}$ onto the second summand, $\sum K_{\mu}+\sum G_{\mu}$. Set $T=\pi \phi\left(\left(\sum K_{\mu}+\sum G_{\mu}\right)[p]\right)$. Then

$$
\phi\left(\sum_{I_{\lambda+1}} G_{\mu}[p]\right)=H[p]+T .
$$

Furthermore, $\pi \phi$ is a projection of $\left(\sum_{I_{\lambda}} K_{\mu}+\sum_{I_{\lambda+1}-I_{\lambda}} G_{\mu}\right)[p]$ that does not decrease heights; once again by the induction hypothesis, $T$ supports a direct summand $L_{\lambda}$ of $\sum_{I_{\lambda}} K_{\mu}+\sum_{I_{\lambda+1}-I_{\lambda}} G_{\mu}$ which is a direct sum of no more than $\aleph_{0}\left|I_{\lambda+1}\right|$ closed groups. It follows that $L=\sum_{\lambda \in \Lambda} L_{\lambda}$ is a pure subgroup of $G$ supported by $S$. A close analysis of the proof of Theorem 1 reveals that $L$ is a direct summand of $G$. Since any direct summand of $G$ supported by $S$ is isomorphic to $L$, the theorem is proved.

Corollary 3. A direct summand of a direct sum of closed groups is necessarily a direct sum of closed groups.

The theorem by Enochs cited earlier and Corollary 3 prove

THEOREM 4. If $G$ is a direct sum of closed groups, then any two direct decompositions of $G$ have isomorphic refinements.

\section{REFERENCES}

1. E. Enochs, Isomorphic refinements of decompositions of a primary group into closed groups, Bull. Soc. Math. France 91 (1963), 63-75.

2. L. Fuchs, Abelian groups, Pergamon, Oxford, 1960.

3. P. Hill, "Certain pure subgroups of primary groups," in Topics in abelian groups, Scott, Foresman and Co., Chicago, Ill., 1963.

4. - Pure subgroups having prescribed subsocles, Bull. Amer. Math. Soc. 71 (1965), 608-609. 
5. - A classification of direct sums of closed groups, Acta Math. Acad. Sci. Hungar. 17 (1966), 263-266.

6. - Sum of countable primary groups, Proc. Amer. Math. Soc. 17 (1966), 1469-1473.

7. P. Hill and C. Megibben, On primary groups with countable basic subgroups, Trans. Amer. Math. Soc. 124 (1966), 49-59.

8. J. Irwin, F. Richman and E. A. Walker, Countable direct sums of torsion complete groups, Proc. Amer. Math. Soc. 17 (1966), 763-767.

9. G. Kolettis, Semi-complete primary abelian groups, Proc. Amer. Math. Soc. 11 (1960), 200-205.

10. L. Kulikov, On the theory of abelian groups of arbitrary cardinal number, Mat. Sb. 16 (1945), 129-162. (Russian)

11. C. Megibben, A note on a paper of Bernard Charles, Bull. Soc. Math. France 91 (1963), 453-454.

UNIVERSITY OF HousTON 